\title{
Emergency responses to teaching, assessment and student support during the COVID-19 pandemic
}

\author{
Alison Reedy, Kalie Carmichael and Oriel Kelly
}

Under COVID-19 conditions higher education institutions responded rapidly with technological solutions to maintain teaching and assessment for students and to adjust support mechanisms to compensate for the lack of face-to-face interaction with students. Using critical reflection as a research method, this paper captures the experiences of three education developers from different institutions to business as usual in the context of emergency remote teaching. The authors utilise the Substitution, Augmentation, Modification, and Redefinition (SAMR) model of technology integration as a conceptual frame to compare their institutions' approaches to educational delivery during the pandemic. Additionally, the authors reflect on student diversity, inclusion and equity during the period of emergency remote teaching.

Keywords: Emergency remote teaching, equity, educational design, education developers, student support, SAMR Framework.

\section{Introduction}

In the scramble to maintain learning continuity and student progression and success under COVID-19 conditions, Australian and New Zealand tertiary institutions relied on technological solutions for teaching, assessment and student learning support (UNESCO, 2020a). Three education developers explored the emergency responses to the COVID-19 pandemic at their respective institutions. This exploration was guided by the question - What are the similarities and differences in the three institutions' emergency responses to teaching and learning during the pandemic? Each author was impacted by COVID-19 as institutional priorities shifted and changed. This paper captures the authors' three main work foci during this time: the scramble from face-to-face to online teaching; the transition from face-to-face invigilated examinations to online exams or alternative assessments; and, revised approaches to supporting student learning during times of pandemic.

\section{The changing teaching and learning context in response to COVID-19}

The exploration shared in this paper represents a microcosm of the changes taking place in higher education in response to the COVID-19 pandemic, inclusive of the reshaping of higher education (Ross, 2020) and the shifting roles of education developers, with many stepping up to drive institutional change agendas in "authentic systems of shared leadership enabling local decision making" (Fernandez \& Shaw, 2020, p. 40). Education developers have been engaged in rapid "transformative learning... and serious adaptive work" (Fernandez \& Shaw, 2020, p. 40), whilst also supporting others. Paradoxically, at a time when education developers are increasingly needed and valued (Ross, 2020), higher education academics and professionals face increased job uncertainty as unemployment has surged (Blustein et al., 2020).

As a response to the COVID-19 pandemic, many Australian and New Zealand higher education institutions moved to online models of teaching and assessment or rapidly altered existing assessment and support mechanisms. This swift pivot to online has been coined as 'emergency remote teaching' to distinguish it from planned, designed and high-quality online teaching (Hodges, Moore, Lockee, Trust \& Bond, 2020). The speed of the response by academic leaders to this emergency as face-to-face teaching, assessment and support became untenable, meant that there was little time "to think about meta concepts like design thinking, or great user experience (UX) design. Rather, in the short-term, [the response needed] to be crude, efficient and responsive" (Sankey, 2020). This is reflected in the direct substitution of face-to-face pedagogies into online environments without functional change or transformation, such as the replication of face-to-face examinations online.

For students, the move from face-to-face to remote and online delivery has resulted in many experiencing disconnection from their peers and the academic community. This may result in deferrals, applying at another 
institution, or withdrawal (Akuhata-Huntington, 2020). To reduce educational disconnection Kaur, Noman and Nordin (2017) encourage academics to engage in inclusive practices in collaboration with students and to reexamine inclusive practices by shifting the question from How do we include students? to How do we make students feel included? Inclusive practice in the shift to online learning must address the digital divide and the impact on students who do not have consistent or adequate access to appropriate technologies. AkuhataHuntington (2020) highlights that 1 in 4 students had limited to inadequate access to technologies in the shift to online learning and $55 \%$ of students experienced a negative online learning journey. Inclusion in online learning is based on the tacit understanding that students have the knowledge and skills to engage in online learning and understand the changed expectations and social conventions of the virtual learning environment (VLE). Making students feel included requires explicit communication of structures, guidelines, course plans and social conventions (Blasco, 2014) so that students are equipped to engage successfully in online learning.

Academic leadership is a requirement for pedagogical transformation (Fernandez \& Shaw, 2020). The skills and knowledge of education developers are critical in leading pedagogical change, making them highly desirable as change leaders for the foreseeable future (Ross, 2020). In times of crisis, it is distributed situational leadership that allows for greater "agility, innovation, and collaboration... than is possible in institutions clinging to an outdated and inflexible hierarchical leadership paradigm" (Fernandez \& Shaw, 2020, p. 40). In the authors' institutions, education developers stepped up during the pandemic and led from the coal-face, moving quickly to support academics by providing training, support, resources and strategies for the 'new normal'. This included asking questions about how the new normal encompasses diversity and inclusion for different student cohorts given that the pandemic is "exacerbating the huge social inequalities that already existed" (Benach, 2020). Such questions probe the impact of the digital divide on students during the pandemic and challenge the role of higher education in reinforcing dominant culture norms and reproducing them online (Hando, 2014).

\section{SAMR as a Conceptual Frame}

In this paper we use Puentedura's (2006) Substitution, Augmentation, Modification, and Redefinition (SAMR) model of technology integration as a conceptual frame to describe and categorise the sophistication of the three institutions' emergency remote teaching responses. In the SAMR model technology integration is considered to be a continuum that moves from pedagogical enhancement (substitution and augmentation), to pedagogical transformation (modification and redefinition). For many educators worldwide who engaged in the COVID-19 induced "experiment in remote learning" (UNESCO, 2020), their first attempts at the redesign of tasks using technology tools sat at the lower end of the SAMR continuum. However, while the substitution response prevailed, the rapid nature of the response that educational developers, academics and institutions made to the changed educational environment was "counter to the perception that changes in academia occur only at glacial speed!” (Fernandez \& Shaw, 2020, p. 40). It demonstrates that educators can quickly adapt to new contexts to ensure the continuity of student learning. The conceptual frame of the SAMR model allows us to envision what this new normal could look like, with the substitution model providing a stepping stone towards the redefinition of education where technology integration is combined with transformative pedagogical approaches.

\section{Research Design}

In the exploration of institutional responses to teaching and learning during COVID-19, the authors used critical reflection as a research method (Fook, 2011), where critical reflection is understood "as a way of learning from and reworking experience" (Fook, 2011, p. 56). The authors shared stories of their changing work foci and roles during the emergency teaching response to COVID-19 and examined the "fundamental assumptions ... to do with power and connections between the individual and the social context[s]" (Fook, 2011, p. 56) in which they were operating. Through shared exploration of these experiences the authors identified similarities and differences in their institutions' responses which led to reconstruction of their professional identities.

\section{Findings}

The findings of this study are represented as three key overlapping areas of teaching and learning focus during the emergency phase of COVID-19. These are: (1) the shift from face-to-face to online teaching; (2) the transition from face-to-face invigilated examinations to online exams or alternative assessments; and, (3) revised approaches to supporting student learning during times of pandemic. 


\section{Scaling up to online teaching}

When the pandemic took hold in early 2020, two of the three authors were education developers at institutions that were well developed as online providers of higher education. This contrasted with the experience of the other author who worked at an institution where face-to-face teaching was the norm (Institution A). While there was little change in the support provided around online teaching at the first two institutions, at Institution A there were significant changes in workload and associated stress for the education developer.

In response to the pandemic, Institution A moved to remote online learning in mid Semester 1 2020. Prior to a formal decision made by the institution to move online, academic staff had been monitoring the pandemic and had begun preparing their units for online remote delivery. The changes required to move online were immense. To enable the emergency response to online teaching, staff were given some time for self-directed upskilling to inform use of online-learning tools within the VLE. The education design team provided academic drop-in educational technology sessions and one-to-one appointments. These provided support for academic staff in shifting lectures and tutorials online, resourcing quick and sustainable remote learning materials, and making adjustments to assessment tasks for online delivery. This shift required tremendous time and effort from the education design team, involving adaptation of business as usual services, such as course and unit design workshops into online formats. Institution A's approach considered the timing and urgency of moving online to assure student continuity of learning and mitigate against the potential retention risks of an abrupt cessation of study while redesign and remote learning applications were implemented.

However, there was minimal pedagogical innovation and little to no functional improvement as a result of technology integration, which had consequences for students. The shift to online remote delivery focused on providing students with an expedited exchange of knowledge and some skills, via the transmission of static content but did not explicitly support student diversity, inclusive pedagogical practices or equity. Although Institution A ensured that its library on campus would remain open for students to access computers, further accommodations are needed to ensure equitable learning opportunities for students with limited access to technology, including access to offline learning material and transformative offline learning experiences.

\section{Moving exams online or to alternative assessment formats}

The pandemic impacted on assessment practices at the authors' institutions. At Institutions A and B, invigilated face-to-face exams were moved online or changed to alternate forms of assessment. At Institution A, online proctoring software was used with online exams, whereas Institution B opted not to use online proctoring. Decision-making about online examinations balanced concerns about privacy and the impact on students of the digital divide with those of exam integrity and professional body requirements. At Institution C, exams were not used but existing online assessments were redesigned where practice-based skills and knowledge were assessed.

For Institution A, online proctored examinations were considered a priority for units that had professional accreditation requirements. Proctoring was implemented in conjunction with the redesign of examination questions to address academic integrity concerns and focus assessment on high-level thought processes and applied problem solving. An additional 10 minutes was allocated to students in the examinations for access and set-up. Students had access to a practice examination to familiarise themselves with the software, however, this could not account for all types of examination settings available. For consistency, academic staff were encouraged to use specific settings in the proctoring software, though with some flexibility. An exam support team was allocated to each proctored exam in the virtual room of each unit to triage questions and address technical complications. The success of troubleshooting varied and was impacted by factors such as random timeouts as well as students' bandwidth and digital navigation skills needed to access the available help.

At Institution B, online exams were not proctored due to equity concerns related to the additional financial and technological burden proctoring may impose on students. Across the institution the online exams were centrally timetabled, and discipline specific approaches were taken to examination formats. Where giving students extra time did not raise academic integrity concerns, up to one additional hour was added to exams to allow for download and upload of documents and to accommodate any technical glitches. In the case of exams deployed in the online test tool, which were mainly in multiple choice format, exams were tightly timed, and questions and answers were randomised to minimise opportunities for collusion. 
At Institution C, existing authentic, practice-based online assessments were quickly enhanced or redesigned. The assessment tasks affected were those with realism, contextualisation and problemisation in their design (Raymond, Homer, Smith \& Grey, 2013). These involved, for example, the application of theory to a real event selected for analysis. Substitute tasks based on hypothetical situations were introduced in order not to penalise students in applied degrees who, due to the pandemic, did not have access to face-to-face interactions or group or praxis situations upon which to base their critical analysis. The educational developer advised on the validity of changes in assessments and the requirement for extra explanation in instructions to enable rapid deployment and ensure equity for the affected cohort. Advice was also provided to the faculty on how to design just-in-time, virtual, personal support to students.

\section{Student support during COVID-19}

Ordinarily, education developers do not work directly with students but support staff to support students. At institution $\mathrm{A}$, this did not change. In contrast, at institution $\mathrm{B}$, the educational developer had a significant change in role that involved consulting with students on exam format design and supporting them on the help desk during the un-proctored online exams. At Institution $\mathrm{C}$, once campuses closed and students no longer had centralised access to faculty, all support mechanisms for teaching and assessment purposes shifted online, with extensive use of email, chat, phone, Zoom sessions and Q\&A forums. Previously mandated face-to-face teaching, which would have particularly benefited diverse student groups, were cancelled. These were substituted with online filing cabinet repositories of resources. This swift response allowed students to autonomously continue studying, although effective support relies on appropriate teacher presence in the online environment (Stone \& Springer, 2019) rather than just providing access to resources. Teacher presence encompasses methods used to create quality learning experiences that support and sustain productive communities of inquiry, consisting of three dimensions: (1) design and organisation, (most often the work of education developers); (2) facilitating discourse; and (3) direct instruction (Garrison, Anderson \& Archer, 2000). While no additional professional development was offered to staff at Institution C, faculty were reminded by the education developer of the outcomes of previous training sessions on 'standing by', that is, of understanding when to step in, to support or directly teach and provide practical support for learning and assessment activities.

\section{Discussion}

In the emergency response to the pandemic, each of the three institutions was forced to rapidly respond to the changed educational environment. While each of the institutions was at different stages of technology integration in terms of their overall teaching and learning approaches, they all had to make emergency changes to aspects of their operations. These changes to teaching, assessment and student support largely fit within the substitution and augmentation stages of the SAMR model. That is, changes such as making static resources available to students via the VLE and running online proctored exams reflect pedagogical change rather than pedagogical transformation. Moving past the emergency response and into the new educational normal, we need to consider if greater technology integration as per the SAMR model provides a vision for the future through the 'modification' of teaching and assessment tasks with significant redesign or through 'redefinition' of novel tasks made possible because of technology (Puentedura, 2006).

However, we need to remain wary that technology integration is not inherently positive and is influenced by factors such as context (Hamilton, Rosenberg \& Akcaoglu, 2016). Because of COVID-19, technology integration has become integral to higher education teaching and student learning. In the rapid transition to online learning, many students reported being unprepared for this mode of engagement (Akuhata-Huntington, 2020). This speaks to the issue of inequity emerging from the emergency response, particularly in relation to the digital divide. It is likely that the most impacted by the transition are students from equity groups, students from culturally diverse backgrounds (Vail, 2019), international students, and those who are older, or first in family (Stone \& O'Shea, 2019). This is a reminder that in the new normal, decisions about the extent of technology integration need to be taken hand in hand with consideration of who will be impacted and how.

Change-decisions at the authors' institutions at the start of the pandemic took student diversity into account in different ways, such as in the decision by Institution B not to embrace online proctoring because of the potential financial and technological burden it placed on students. As we plan for the new normal, it is incumbent on education developers to consider how inclusiveness has been impacted by the emergency response measures, and to place student diversity at the centre of learning and assessment design decisions. Should we wish to 
enable transformative learning experiences (Mezirow, 1991), our learning design decisions must acknowledge the barriers created by the digital divide and dominant culture. The pandemic has necessitated a rethinking of how we 'do' higher education. This provides an opportunity for the modification and redefinition of learning at our institutions to embrace the diversity of our student cohorts in our educational design decision making.

\section{Conclusion}

During the scramble to fully online learning in Semester 12020 , the urgency for universities to maintain an educational presence outweighed the ability of education developers to support fully formed learning design processes. The initial educational response to the pandemic of emergency remote teaching has now shifted at the authors' institutions to a review of the effectiveness of those responses and evaluation of appropriate and sustainable evidence-based practice for what appears to be the new normal of socially distanced education. Beyond the emergency response, the role of education developers in our three institutions has shifted, though our roles as change leaders continues as we to support educators to evaluate pedagogical processes, identify sustainable improvement, and re-design learning that is fit for purpose. This involves the modification and redefinition of teaching and learning that integrates pedagogical and technological innovation (Puentedura, 2015 ) with inclusive practices that aim to deliver equitable outcomes for our diverse student populations.

\section{References}

Akuhata-Huntington, Z. (2020). Impacts of COVID-19 lockdown on Māori university students. Wellington: Te Mana Akonga. https://www.temanaakonga.org.nz/nga-puka

Benach, J. (2020, May 6). The pandemic kills the poor: Inequality will kill them even more. The Bullet. https://socialistproject.ca/2020/05/pandemic-kills-the-poor-inequality-will-kill-more/

Blasco, M. (2014). Making the tacit explicit: Rethinking culturally inclusive pedagogy in international student academic adaptation. Pedagogy, Culture \& Society, 23(1), 85-106. https://doi.org/10.1080/14681366.2014.922120

Blustein, D.L., Duffy, R., Ferreira, J.A., Cohen-Scali, V., Cinamon, R.G., \& Allan, B.A. (2020). Unemployment in the time of COVID-19: A research agenda. Journal of Vocational Behaviour, 119(2020), 103436, 1-4. https://doi.org/10.1016/j.jvb.2020.103436

Fernandez, A.A. \& Shaw, G.P. (2020). Academic leadership in a time of crisis: The coronavirus and COVID-19. Journal of Leadership Studies, 14(1), p. 39-45. https://doi.org/10.1002/jls.21684

Fook J. (2011). Developing critical reflection as a research method. In: Higgs J., Titchen A., Horsfall D., Bridges D. (eds). Creative Spaces for Qualitative Researching. Practice, Education, Work and Society, vol 5. Sense Publishers. https://doi.org/10.1007/978-94-6091-761-5_6

Garrison, D.R., Anderson, T., \& Archer, W. (2000). Critical inquiry in a text-based environment: Computer conferencing in higher education. The Internet and Higher Education, 2(2-3), 87-105. https://doi.org/10.1016/S1096-7516(00)00016-6

Hamilton, E.R., Rosenberg, J.M. \& Akcaoglu, M. (2016). The substitution augmentation modification redefinition (SAMR) model: A critical review and suggestions for its use. TechTrends, 60, p. 433-441. https://doi.org/10.1007/s11528-016-0091-y

Hando, A. (2014). Does cultural self-awareness alter instructional design decisions? (Doctoral dissertation). https://researchrepository.wvu.edu/etd/645/

Hodges, C., Moore, S., Lockee, B., Trust, T. \& Bond, A. (2020, March 27). The difference between emergency remote teaching and online learning. Educause Review. https://er.educause.edu/articles/2020/3/thedifference-between-emergency-remote-teaching-and-online-learning

Kaur, A., Noman, M., \& Nordin, H. (2017). Inclusive assessment for linguistically diverse learners in higher education. Assessment \& Evaluation in Higher Education, 42(5), 756-771. https://doi.org/10.1080/02602938.2016.1187250

Mezirow, J. (1991). Transformative dimensions of adult learning. Jossey-Bass, 350 Sansome Street, San Francisco, CA 94104-1310.

Puentedura, R. (2006). Transformation, technology, and education [blog post]. http://hippasus.com/resources/tte/

Raymond, J., Homer, C., Smith, R., \& Gray, J. (2013). Learning through authentic assessment. An evaluation of a new development in the undergraduate midwifery curriculum. Nurse Educational and Practice, 13(5), 471-476. https://doi.org/10.1016/j.nepr.2012.10.006 
Ross, J. (2020, May 8). COVID crisis could see 'learning designers' supplant academic roles. Times Higher Education World Institution Rankings. https://www.timeshighereducation.com/news/covid-crisis-could-seelearning-designers-supplant-academic-roles

Sankey, M. (2020, March 16). Teaching online in the present crisis? What you need to know now. Campus Morning Mail. https://campusmorningmail.com.au/news/teaching-on-line-in-the-present-crisis-what-youneed-to-know-now/?utm_campaign=website\&utm_source=sendgrid.com\&utm_medium=email

Stone, C. \& O'Shea, S. (2019). Older, online and first: recommendations for retention and success. Australasian Journal of Educational Technology, 34(5), 57-69. https://doi.org/10.14742/ajet.3913

Stone, C. \& Springer M. (2019). Interactivity, connectedness and "teacher presence": Engaging and retaining students online. Australian Journal of Adult Learning, 59(2), 146-169. https://files.eric.ed.gov/fulltext/EJ1235966.pdf

UNESCO. (2020, April 10). Exams and assessments in COVID-19 crisis: Fairness at the centre. UNESCO. https://en.unesco.org/news/exams-and-assessments-covid-19-crisis-fairness-centre

Vail, H. (2019). Examining the need for culturally responsive digital learning. Waikato Journal of Education, 23(2), 17-23. https://doi.org/10.15663/wje.v23i2.652

Reedy, A., Carmichael, K. \& Kelly, O. (2020). Emergency responses to teaching, assessment and student support during the COVID-19 pandemic. In S. Gregory, S. Warburton, \& M. Parkes (Eds.), ASCILITE's First Virtual Conference. Proceedings ASCILITE 2020 in Armidale (pp. 246-251). https://doi.org/10.14742/ascilite2020.0138

Note: All published papers are refereed, having undergone a double-blind peer-review process.

The author(s) assign a Creative Commons by attribution licence enabling others to distribute, remix, tweak, and build upon their work, even commercially, as long as credit is given to the author(s) for the original creation.

(C) Reedy, A., Carmichael, K. \& Kelly, O. 2020 